\title{
Consumo de alimentos en el Colegio y Universidad de Santo Tomás de Aquino, 1733-1755*
}

Food Consumption at the Colegio and Universidad Santo Tomás de Aquino, I733-I755

DOI: https://doi.org/10.22380/20274688.1145

Recibido: 21 de enero del 2020

Aprobado: 9 de septiembre del 2020
RAMIRO ALONSO SÁNCHEZ CORAL**

Universidad del Tolima, Ibagué, Colombia rasanchezc@ut.edu.co

\section{R E S U M E N}

El objetivo de este artículo es estudiar algunos aspectos del consumo de alimentos en el Colegio y Universidad de Santo Tomás de Aquino en la ciudad de Santafé, entre 1733-1737 y 1751-1755. Este trabajo pretende abordar de manera central la información relacionada con el consumo de alimentos que eran comprados y que se encuentran registrados en el libro de gastos de la institución. El estudio presenta dos constataciones significativas para el estudio del consumo y la vida cotidiana en el Nuevo Reino de Granada: la primera, sobre la composición detallada del gasto en alimentos registrado en los libros contables de una comunidad educativa religiosa; en segundo lugar, que la frecuencia y el consumo de algunos pro-

* Este artículo es resultado del proyecto de investigación "El consumo eclesiástico y la circulación de productos en tres ciudades de Nueva Granada: Santafé, Popayán y Santafé de Antioquia, 1730176I", código: I40I18, financiado por la Oficina de Investigaciones y Desarrollo Científico de la Universidad del Tolima, Ibagué, Colombia.

** Historiador de la Universidad Nacional de Colombia, sede Bogotá y magíster en Historia de la Universidad de los Andes, Bogotá, Colombia. Se desempeña como investigador de la Biblioteca Virtual del Banco de la República y profesor de la Universidad del Rosario y de la Universidad del Tolima. Hace parte del grupo de investigación Hechos - Historia Económica y Social, avalado por Colciencias (categoría C). 
ductos específicos no dependían solo de las posibilidades del mercado, sino también del contexto cultural institucional, siendo uno de los aspectos más importantes el marco de creencias religiosas de la orden de los dominicos.

Palabras clave: consumo de alimentos, Nuevo Reino de Granada, Santafé, siglo xVIII, Colegio Mayor de Santo Tomás, Orden de Predicadores

\section{$\begin{array}{llllllllllllllllll}\mathbf{A} & \mathbf{B} & \mathbf{S} & \mathbf{T} & \mathbf{R} & \mathbf{A} & \mathbf{C} & \mathbf{T}\end{array}$}

The main objective of this article is to study some aspects of food consumption at the Colegio y Universidad Santo Tomás de Aquino in Santafé, between 1733-1737 and 1751-1755. This work aims to analyze both the information related to the food consumption and the food purchased that was registered in the Institution's expense book. The study presents two significant findings for the study of consumption and daily life in the Nuevo Reino de Granada.
The first one, about the composition of food expenditure registered in the accounting books of a religious educational community; the second one, that the frequency and consumption of some specific products did not depend only on the possibilities of the market, but also on the institutional cultural context, where one of the most important aspects was the religious belief framework of the Order of Preachers (Dominican Order).

Keywords: food consumption, Nuevo Reino de Granada, Santafé, 18th Century, Colegio and Universidad Santo Tomás de Aquino, Dominican Order

\section{Introducción}

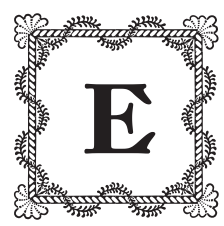

1 crecimiento institucional educativo de la Orden de Predicadores se dio con la fundación del Colegio de Santo Tomás de Aquino, establecido en Santafé a inicios del siglo Xvir. La fundación correspondía a la obra pía que quiso dejar Gaspar Núñez a favor de la orden ${ }^{1}$. El colegio fue incorporado rápidamente a esta última el II de julio de I608, "con la condición de pedirle a su Santidad el traslado del título de universidad que ostentaba el Estudio General del Convento de Nuestra Señora del Rosario al Colegio de Santo Tomás" (Plata y Reyes Ioo), traspaso que fue autorizado cuatro años después por Paulo V (Pacheco 560). La figura de universidad le permitiría a la orden establecerse como institución educativa

I En su testamentaria Núñez prometió 30000 pesos de oro de iz quilates para la fundación de un colegio con el nombre de Santo Tomás de Aquino (Plata y Reyes 98). 
con los máximos niveles de estudio, además de tener la posibilidad de otorgar grados, recibir regalías reales y vincularse a las redes de poder más importantes en el Nuevo Reino de Granada por medio de sus estudiantes.

Sin embargo, la lucha por la monopolización del contexto educativo llevó a una competencia entre la Orden de Predicadores y la Compañía de Jesús. Esta lucha entre las órdenes llevaría a una serie de demandas y procesos legales que durarían todo el siglo Xvir y que impedirían que ambas comunidades lograran consolidarse como universidades en ese siglo². Pese a ese contexto de pleitos, el Colegio y Universidad de Santo Tomás (Cust de ahora en adelante) logró ganar algunos reconocimientos. El Consejo de Indias falló a favor de los dominicos en torno al legado de Núñez, legitimó su estatus por medio de una bula y breves pontificios a partir de I6I2 (AGN, C, C, I, ff. 419 r.-422 v.) y, además, logró compartir las facultades de otorgar grados en las mismas áreas y periodos que le habían autorizado a los jesuitas. Finalmente, según los dictámenes del papa Clemente XI y por decreto del 3 agosto de I70I, la Corona dictaminaría el estatus final de la educación en el Nuevo Reino de Granada: tanto el CUsT $^{3}$ como la Academia Javeriana tendrían los mismos derechos y posibilidades de otorgar grados en Santafé (Plata y Reyes iı6; Pacheco 563). En síntesis, la batalla por el monopolio de la educación en el Nuevo Reino de Granada había terminado en tablas para ambas instituciones.

Los estudios que desde su fundación ofreció el cust se relacionaron con lo que importaron los dominicos (Bernadot 190) como currículo de estudios generales,

[...] diseńado en 1259 por Alberto Magno y Tomás de Aquino, y con la que organizaron las primeras universidades coloniales [...] Y consistían en estudios de artes o filosofía durante tres o cuatro ańos. Trienio de filosofía en el que se estudiaba dialéctica, filosofía y metafísica; estudios de Teología, que duraban entre cuatro y seis ańos y donde era obligatorio el estudio de la Summa Teológica de Santo Tomás, y el estudio de

2 Según Plata y Reyes, entre i608 y r 70 r los jesuitas impugnaron la fundación del Colegio de Santo Tomás, reclamaron derechos sobre la herencia de Gaspar Núñez, solicitaron facultades para otorgar grados durante diez años en Filipinas, Chile, Tucumán y el Nuevo Reino de Granada y recusaron la condición de universidad del cust y las aprobaciones pontificias y reales de su funcionamiento (IO-IO4 y III-II2).

3 La estructura organizacional del CUST estuvo compuesta por: rector, vicerrector, regente de estudios, maestro de estudiantes, un catedrático para cada disciplina (teología, artes, gramática, lengua indígena, medicina) y cuatro colegiales (Plata y Reyes ı०9-IIo). 
la sagrada escritura, lenguas hebrea y griega (para el caso europeo) y en América colonial se encontraba relacionado con las lenguas indígenas. (Plata y Reyes 76-77)

El funcionamiento del cust se guiaba por el régimen de castas y las constituciones excluían a cualquiera que no demostrara con suficiencia su "limpieza de sangre”. Solo existían algunas excepciones vinculadas a los indígenas que fueran descendientes de caciques prominentes y algunos individuos acaudalados (Plata y Reyes 68-69). La mayor parte de los cargos eran ocupados por miembros de la Orden de Predicadores. La estructura de ingresos del cust en la primera mitad del siglo XVIII contaba con los sistemas tradicionales de financiamiento de las órdenes religiosas, es decir, haciendas, censos, donaciones, obras pías, capellanías, cofradías y, de manera adicional, los ingresos por concepto de derechos de grados relacionados con sus actividades formativas.

Una de las responsabilidades delegadas a los rectores fue el manejo de las cuentas de manutención. Los padres debían llevar libros de gastos y estos contenían, entre otras informaciones, aquella relativa a las compras de alimentos, enseres y objetos para el uso y el consumo de los colegiales. Al revisar las Constituciones de los padres dominicos para el Colegio de Nuestra Señora del Rosario, que se inspiraron en las del Colegio de Salamanca, podemos observar, por ejemplo, regulaciones más específicas en los temas de consumo de alimentos:

Constitución IX: Disponemos, que sean tratados con toda dezencia los collegiales, y combitores en la comida: y que su ordinario sea algún asado por principio, o de tocino o de lomo, o de cabrito, que luego se les dé, o gigote ${ }^{4}$ de carnero o albóndigas, o pastel en vote, o cosa semejantes, lo tercero, la olla con baca y carnero, con tocino, y repollo: y lo último postre de algún dulce de trapiche, o queso, o cosa semejante, y los días de capilla se les añada un cuarto de ave, o conejos, o tórtolas, o perdices, que pareze que basta para el regalo decente con templanza christiana. Y a la cena algún gigote, o ajiacos con los mismos postres.

4 Se trata de un guiso con una proteína picada, podía ser de carnero, ternera o inclusive de gallina, que tenía como base principal de sazón la manteca de cerdo y diferentes especias (Diccionario, t. IV (1734). Véase http://web.frl.es/DA.html).

5 El ajiaco es una sopa espesa que contiene diferentes clases de papas (amarilla o criolla, sabanera y papa pastusa), mazorca, hierbas, pollo y condimentos. El origen de este plato se encuentra en la sabana de Santafé como resultado del mestizaje gastronómico entre los productos indígenas (papas, 
Más los viernes y días de quaresma se les dará un par de huevos, y un guisado de garvanzos, alverjas, o habas, dos pescados, arroz y postre a comer, y lo mismo el sábado. Más el viernes no se les dé de cenar, sino algunas hervas aderezadas, y algún postre de dulce: los sabados se les podrá dar de cenar algunas hervas, una tortilla de huevos y su postre. (AHUR, $C, 4, \mathrm{f} .3 \mathrm{v}$. $)^{6}$

Gran parte del consumo de alimentos en el cust era producto de la adquisición en el mercado, pero otros productos se encuentran relacionados con otros canales de abastecimiento, vinculados con las obligaciones de la Corona en cumplimiento del Patronato Regio o por conducto de las propias haciendas relacionadas con la orden.

Este trabajo busca abordar, de manera central, la información relacionada con el consumo de alimentos que se compraban en el mercado, cuya adquisición se encuentra registrada en el libro de gastos del cust. La información hallada en este libro permite reconstruir parte de esa historia de los alimentos consumidos, poniendo en evidencia no solo los factores de la dimensión económica y la dependencia geográfica del mercado, sino también una concepción de mundo de parte de los miembros de esa comunidad mediante los productos con que se alimentaban en su cotidianidad.

En términos generales, la historiografía colonial del Nuevo Reino de Granada se ha preocupado por esta temática, con una gama relativamente amplia de textos, enfoques e interpretaciones (Saldarriaga, "Consumo"; Saldarriaga, Alimentación; P. F. Vargas; Alzate; Amaya y Torres; Bonnett; Castaño; Pérez; Zambrano; Restrepo). Sin embargo, están por realizarse los estudios sobre el consumo, la reconstrucción de las canastas básicas alimenticias de las instituciones y la comprensión de las razones por las cuales en la Colonia se consumía cierto tipo de productos7. Es probable que ello se explique por problemas relacionados

mazorcas, guascas y sal) y los productos introducidos por los europeos (cebolla, ajo, pimienta, pollo, alcaparras, entre otros).

6 Se han utilizado las Constituciones del Colegio de Nuestra Señora del Rosario por pertenecer a la misma orden. No se utilizaron las que pertenecían al propio CUST porque, como lo mencionan Plata y Reyes, estas se destruyeron con otra documentación de la institución en el incendio de 176 I, o se perdieron en los litigios ( 122 y I26).

7 Estas avanzaron en la caracterización de la alimentación de la sociedad neogranadina en los siglos tempranos, las relaciones de la vida cotidiana en torno a la consecución y el consumo de alimentos, y también las relaciones entre alimentación e identidad. Sin embargo, hasta el momento, no se había 
con la escasez documental, la dispersión de los datos y la dificultad para el procesamiento de la información de los libros de gastos ${ }^{8}$.

Esta investigación busca complementar parte de la historia del consumo de alimentos en la sociedad colonial temprana del siglo xviII, mediante el uso de una fuente documental inédita. Es un ejercicio muy preciso que se inserta en los problemas estructurales de la economía y la cultura. En este último aspecto en particular vale la pena retomar una perspectiva amplia y observar, como lo plantearían Mary Douglas y Baron C. Isherwood, que los bienes no son exclusivamente necesarios para la subsistencia, sino que también son necesarios para hacer visibles y estables las categorías de una cultura (74). En concreto, la investigación propone un análisis del gasto diario del cust para los quinquenios de I733-I737 y I75I-I755, una reconstrucción de la canasta de productos consumidos por la institución y la exploración cualitativa de las zonas de procedencia de algunos de los productos alimenticios. El estudio busca responder a dos preguntas fundamentales: ¿cuáles eran los productos consumidos por la comunidad del cust durante los dos quinquenios? Y ¿qué nos permite comprender el libro de gasto diario sobre el consumo de alimentos y algunos patrones de la alimentación de la institución?

\section{Comprar y registrar: los libros de cuentas de gasto diario y extraordinario}

El registro de las compras de alimentos en los libros de gasto diario y extraordinario fue el mecanismo más utilizado por las diferentes órdenes religiosas en el Nuevo Reino de Granada. Los libros fueron usados para la organización de cuentas y el seguimiento de los costos que requerían las instituciones con el

logrado realizar el procesamiento de todo un libro de cuentas de alguna entidad colonial que sea una fuente que permita hacerse un panorama más específico. En ese sentido, consideramos que este ejercicio concreto puede contribuir a los estudios precedentes.

8 Existen diferentes complejidades frente al manejo de este tipo de fuente. Lo primero es la dificultad de encontrar un expediente completo; segundo, sistematizar toda la información de registros semanales durante varios años (en este expediente del cust trabajamos diez años, el documento tenía 233 folios, 466 páginas) que se convirtieron en 3000 registros Excel, pertenecientes a 520 semanas); tercero, uniformizar y normalizar las unidades de medida; y, finalmente, generar las líneas de tendencia, gráficos y análisis. En este sentido, consideramos que esta propuesta constituye un aporte metodológico en la forma que se ha estudiado el fenómeno del consumo de alimentos, siendo tal vez el primer ejercicio de estas características. 
fin de garantizar el desarrollo de sus actividades en los diferentes conventos, colegios, hospitales y expediciones científicas (AGN, SAA-III, $R H-C$, I254C; ACC, OS, 9503, 9504, 9453, 9454, 9609, 9610, 9602, 9600, 9599, 9598, 9590, 9591, 9592, 9594; AHA, C, 457-458; AGN, AI 2; Alzate).

En los libros de gasto se registraron semanal o mensualmente compras de alimentos, de objetos, pagos de salarios y servicios, gastos en festividades y reparaciones. En América Latina este tipo de fuente documental ha permitido recientemente la realización de investigaciones relacionadas con la historia de los precios (Frid; Torres, "El comportamiento"), así como con el análisis de la organización y el funcionamiento de una institución como la Expedición Botánica (Amaya y Torres), o estudios sobre el consumo de alimentos en la sociedad neogranadina (Alzate; Pérez).

Para el presente estudio se ha utilizado una fuente inédita, que no ha sido empleada por la historiografía hasta el momento. Se trata del "Libro de gasto ordinario, extraordinario y recibo del Colegio y Universidad de Santo Tomás de Aquino de la Orden de Predicadores de la Ciudad de Santafé" (agn, SAA-III, $R H-C, \mathrm{I} 254 \mathrm{C})$. Las cuentas registradas en este libro comprenden desde junio de 1732 hasta julio de $1765^{\circ}$, sin embargo, solo hemos trabajado los quinquenios de I733-I737 y I75I-I755, porque son los periodos que contienen información más robusta, en los cuales se presenta una imagen detallada del consumo. Además, es importante recordar - como lo menciona Torres para el caso de los libros de Popayán-que no todos los libros "ofrecen datos continuos de todos los meses [...] los encargados de llevar los registros o bien en ocasiones no especificaban la unidad de medida por dinero invertido o bien hacían un resumen global de los 'gastos de cocina' o 'gasto diario" ("El comportamiento" 636).

\section{Estructura del gasto de la institución}

Los gastos se dividían en "gastos ordinarios" y "gastos extraordinarios". Entre los primeros se encontraban gastos realizados en la compra de alimentos, leña, velas y algunas compras de loza, y dentro de los segundos se encontraban aquellos asociados con compra de loza, manteles, vestuario, el salario del cocinero, fiestas, papel para los colegiales, pagos por algunos trabajos (sacar la basura,

9 Esta fuente también permitiría, por ejemplo, el análisis de la evolución de precios de algunos productos o el estudio de la gestión administrativa de algunos de los rectores del colegio. 
desherbar, entre otros), medicinas y asistencia médica, reparaciones de objetos o infraestructura institucional y algunas compras esporádicas de alimentos que se llevaban a cabo y no se lograba incorporarlas a la sección de gastos ordinarios semanales; sin embargo, no era usual que se registraran compras de alimentos en este "gasto extraordinario", a excepción de dos productos que generalmente ingresaban por esta sección: el cacao y el azúcar (tabla i).

$\rightarrow$ TABLA I.

Muestra de algunos de los gastos extraordinarios realizados en el primer trimestre de I733 en el cUsT

\begin{tabular}{|l|l|c|c|}
\multicolumn{1}{|c|}{ Mes } & \multicolumn{1}{|c|}{ Producto } & Pesos & Reales \\
\hline Enero & Sábanas & 3 & 0 \\
\hline Enero & Cacao & I & 4 \\
\hline Enero & Medicinas y asistencia médica de dos hermanos & 4 & 0 \\
\hline Febrero & Esteras y refacción de celda rectoral & 9 & 4 \\
\hline Febrero & Pago al pintor & 3 & 0 \\
\hline Febrero & $\begin{array}{l}\text { Colocar apóstoles en la capilla, ángeles en el } \\
\text { claustro }\end{array}$ & 3 & 0 \\
\hline Marzo & Fiesta de santo Tomás en la iglesia y refectorio & $2 \mathrm{IO}$ & 0 \\
\hline Marzo & Papel & $\mathrm{I}$ & 0 \\
\hline Marzo & Blanqueamiento de la capilla y claustros & 7 & 0 \\
\hline Abril & Reparación de sillas & $\mathrm{I}$ & 0 \\
\hline Abril & Cacao [dos millares] & 5 & 4 \\
\hline
\end{tabular}

Fuente: elaboración propia a partir de AGN, SAA-III, RH-C, I254C, f. I3I

Las compras de muebles y enseres, las reparaciones de infraestructura y las celebraciones de festividades religiosas eran más costosas que otros gastos. $\mathrm{Al}$ analizar el volumen total de gastos (35 $32 \mathrm{I}$ pesos) realizados entre 1732 y 1765 , se observa que el $45 \%$ (I5 917 pesos) corresponde a "gastos ordinarios", frente a un $55 \%$ (I9 404 pesos) que se registró en los "gastos extraordinarios" (AGN, SAA-III, $R H-C, \mathrm{I} 254 \mathrm{C})$. Teniendo en cuenta que nos interesa el fenómeno relacionado con 
el consumo de alimentos, hemos decidido analizar solamente el "gasto ordinario", pues allí se encuentran la mayoría de los productos de interés. El resto de los productos, objetos, servicios y egresos que constituyen el "gasto extraordinario” escapan del ámbito de análisis de esta investigación.

\section{Composición general del gasto institucional}

El total del gasto en los dos periodos de análisis fue de 5592 pesos, en el periodo de 1733-I735 llegó a 3090 pesos plata y en el periodo I75I-I755 alcanzó los 2502 pesos plata, para un promedio anual de consumo de 617 y 500 pesos, respectivamente. El análisis del gasto de la institución permite realizar tres constataciones importantes en relación con la historiografía. La primera es que los gastos por concepto de alimentos y productos básicos para el funcionamiento del cust no fueron considerablemente altos si se compara con el funcionamiento de otras instituciones coloniales como hospitales o expediciones científicas (Alzate 2223; Amaya y Torres).

La segunda constatación se relaciona con el hecho de que las variaciones encontradas entre ambos quinquenios coinciden con la explicación de James Torres sobre el periodo de crisis y decrecimiento que se produjo entre $1740 \mathrm{y}$ 1750. Torres analiza el comportamiento de los precios en Popayán y Antioquia en relación con las cifras de producción aurífera, tratando de demostrar las conexiones y los niveles de dependencia entre las variables de minería, moneda y economía, y demuestra que el desempeño minero fue inversamente proporcional al de los precios. Este fenómeno, representado mediante el problema inflacionario, llevó a que "desde 1750 hasta I755, se compraba menos con el oro y se invertía más para obtenerlo" ("El comportamiento” 657), situación que terminó afectando las posibilidades de adquisición de productos en la economía del Nuevo Reino de Granada, impacto aún mayor si se tiene en cuenta que buena parte de la producción aurífera antioqueña era negociada en Santafé (Torres, Minería y moneda 46-70).

La última constatación señala que los ingresos de la orden disminuyeron, específicamente por la variación negativa en los recaudos generados por conceptos de derechos de grado, censos y sus réditos correspondientes y otros ingresos de la institución, en particular para los ańos de 1735, I736 y 1750 a 1756 (Plata y Reyes I67-I70), motivo por el cual la inversión total se vio afectada (figura I). 


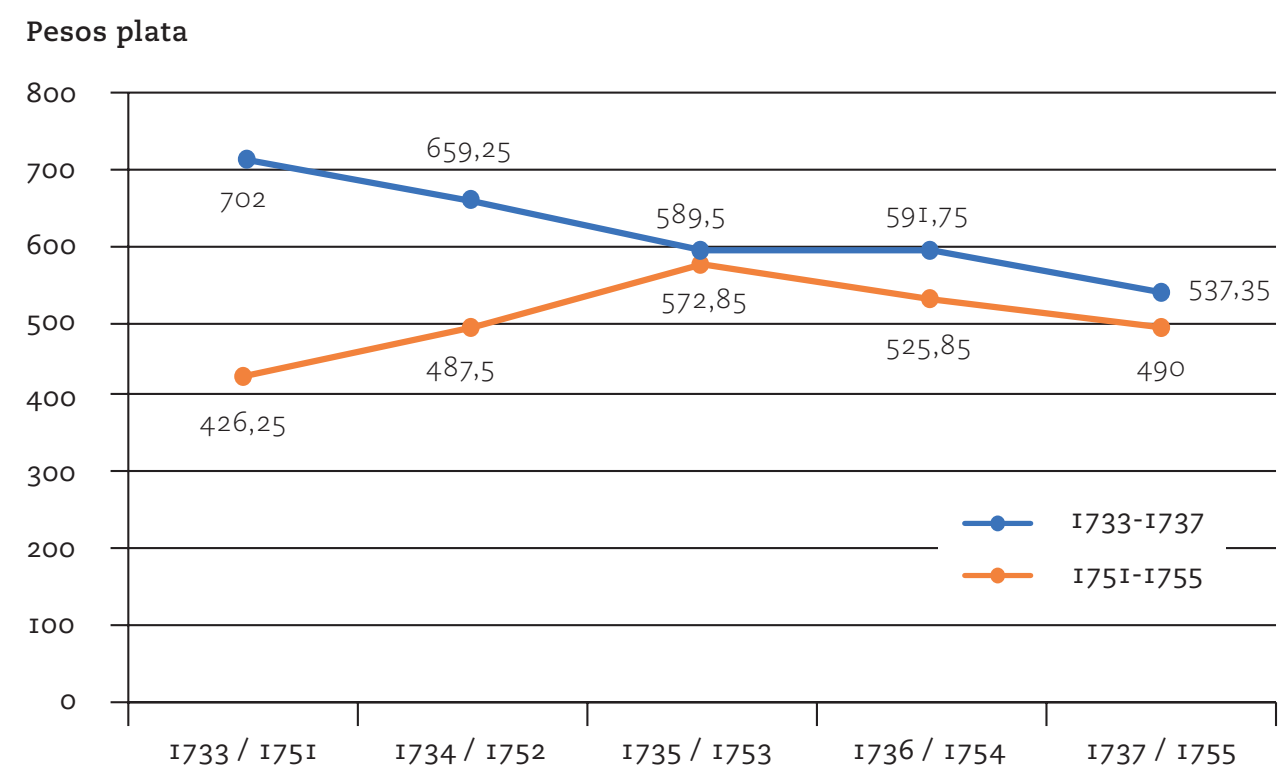

$\rightarrow$ FIGURA I.

Gastos ordinarios del CUST, I733-I737 y I75I-I755

Fuente: elaboración propia con base en AGN, SAA-III, RH-C, I254C

Por lo general, las compras del Cust se realizaban de manera semanal, todos los viernes del mes. Dentro de estos gastos, varios eran los alimentos de adquisición más frecuentes (véase tabla 2), mientras que otros eran comprados solamente en determinados periodos o fechas del año: pescado para la cuaresma, Semana Santa y a veces a fin de año, o gastos adicionales de leche y huevos que se realizaban para la vigilia u otras actividades de carácter religioso. Dentro de ese rubro de "gasto ordinario" se encontraba además un ítem denominado "gasto para la semana" que agrupaba los gastos hechos en la plaza de mercado (AGN, SAA-III, $R H-C$, I254C, f. I8 r., 6I v.). 
$\leftrightarrow$ TABLA 2.

Lista de productos consumidos en el Colegio y Universidad Santo Tomás de Aquino, entre I733-I737 y I75I-I755

\begin{tabular}{|c|c|c|}
\hline \multicolumn{3}{|c|}{ Productos } \\
\hline Pan & Conserva & Carne \\
\hline Papa & Gallinas & Aceite \\
\hline Vino & Cacao & Lomo de puerco \\
\hline Arracachas $^{10}$ & Sal & Costilla de puerco \\
\hline Garbanzos & Ajos & Miel \\
\hline Habas & Cecina & Costilla de vaca \\
\hline Manteca & Huevos & Leche \\
\hline Frijoles & Arroz & Leña \\
\hline Alfandoques & Jamones & Loza \\
\hline Azúcar & Pescado & Velas \\
\hline
\end{tabular}

Fuente: AGN, SAA-III, RH-C, L, I254C

Si bien este registro no se pudo desagregar por completo, de acuerdo con las diferentes anotaciones realizadas por los religiosos se logró determinar que este egreso siempre incluía la compra semanal de los huevos y la leche para la comunidad. Este tipo de registros era frecuente en la contabilidad llevada por las diferentes comunidades religiosas a cargo de los gastos de las instituciones (Alzate 22; Torres, "El comportamiento" 636).

Io La arracacha es un tubérculo originario de los Andes, también conocido como apio criollo o zanahoria blanca, entre otras denominaciones, cuya parte comestible es su raíz. Entre sus valores nutricionales se destacan sus altos niveles de almidón y calcio: "Crece en tierras frías y cuya raíz tuberosa, gruesa y de color amarillo, se come cocida” (Diccionario de la lengua española, http:// dle.rae.es/arracacho\#3eI9m2J). 


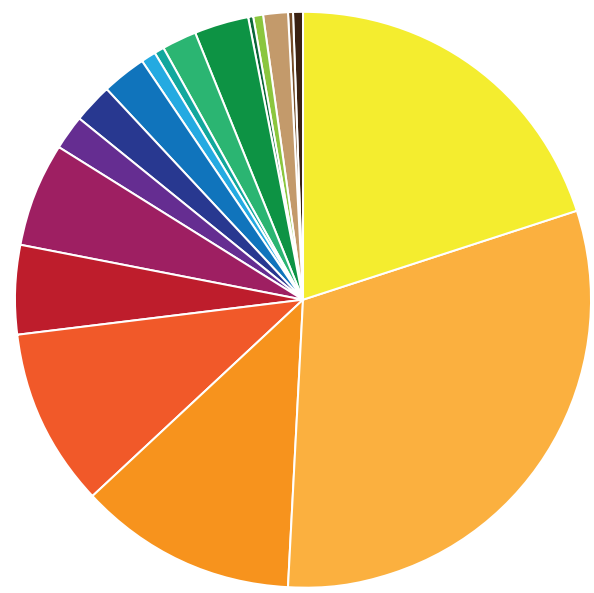

\begin{tabular}{|c|c|}
\hline $\begin{array}{l}\text { Gasto de la } \\
\text { semana } 29 \%\end{array}$ & Garbanzos I\% \\
\hline Pan 3I\% & Loza $0,4 \%$ \\
\hline Turmas I2\% & Alfandoques $2 \%$ \\
\hline Velas I0\% & Leña 3\% \\
\hline Arroz 5\% & $\begin{array}{l}\text { Vigilias y } \\
\text { temporadas 0,5\% }\end{array}$ \\
\hline Manteca 6\% & $\begin{array}{l}\text { Frijoles y } \\
\text { habas } 0,3 \%\end{array}$ \\
\hline Pescado $2 \%$ & Dulce I,6\% \\
\hline Cecina $2 \%$ & Sal $0,3 \%$ \\
\hline Jamones $2,5 \%$ & Otros $0,3 \%$ \\
\hline
\end{tabular}

$\rightarrow$ FIGURA 2 .

Composición porcentual del gasto ordinario del CUST, I733-I737

Fuente: elaboración propia con base en AGN, SAA-III, RH-C,I254C
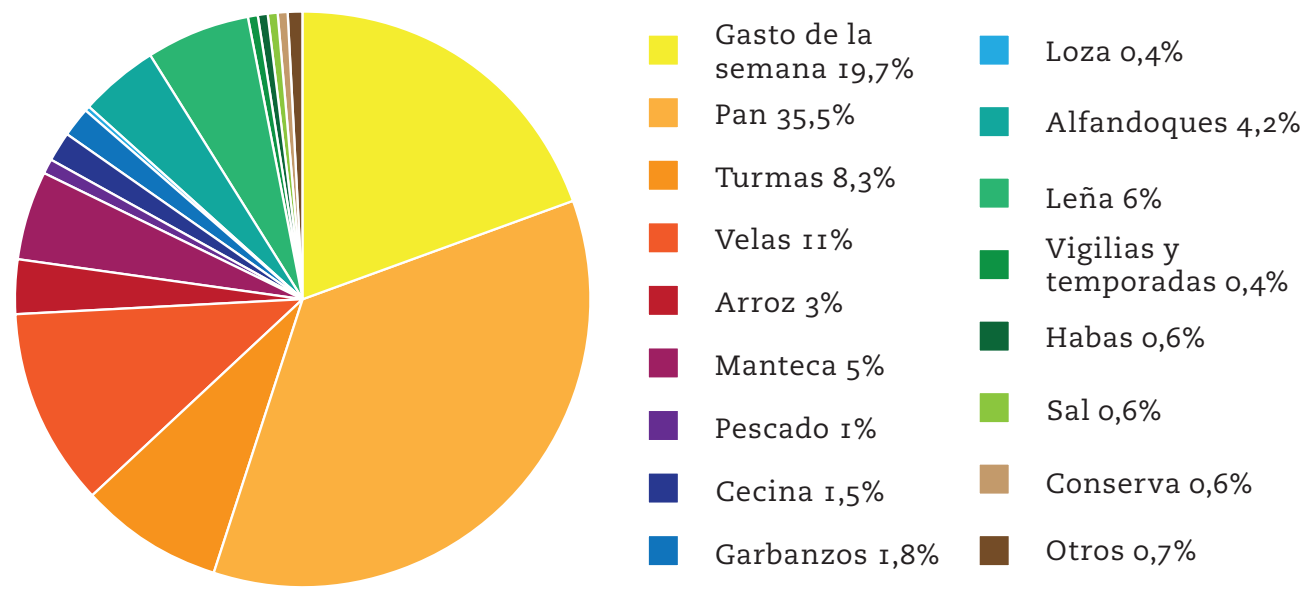

$\leftrightarrow$ FIGURA 3

Composición porcentual del gasto ordinario del CUST, I75I-I755

Fuente: elaboración propia con base en AGN, SAA-III, RH-C,I254C

El gasto más importante que se encuentra en las compras de la institución se asocia con el consumo de pan. Los datos obtenidos muestran que la compra de pan representaba el 3I \% para el primer quinquenio y el 35,5\% para el segundo (figuras 2 y 3). La información registrada en el "gasto diario" no permite conocer las cantidades exactas de pan adquiridas, sin embargo, algunos de los registros 
nos proporcionan información sobre el costo semanal del producto por persona. Por ejemplo, en la última semana del mes de julio de 1737 se menciona "Dimos pan hasta el 3I. Sinco p[eso]s y un real, es a saber a seis colegiales, R[ecto]r, Vic[erecto]r, el h[erman]o lego, y un muchacho, que cocina, a q[uie]n se da un quartillo de pan cada día” (AGN, $R H$-C, SAA-III, I254C, f. I9 v.). A partir de esta información se puede deducir que el consumo de pan por parte de los colegiales y directivos de la institución era de 4 reales y un cuartillo, es decir, dos veces lo que se le asignaba al ayudante de la cocina, a quien diariamente solo se le otorgaba un cuartillo para este producto.

La predilección por el consumo de pan provenía de una amplia tradición europea. En la península, el pan, que tenía un peso muy importante en la dieta de los madrileńos y sevillanos, ocupó el primer lugar en las canastas de compras de alimentos entre finales del siglo Xvir y todo el siglo XVIII (Llopis et al. 39). Esto se explica porque de todos los bienes de consumo, desde muy temprano en el desarrollo de la cultura alimenticia peninsular, el pan se había constituido en el producto de consumo popular más generalizado (Andrés y Lanza) ${ }^{I 1}$. En el Nuevo Reino la adquisición de pan se había establecido desde muy temprano, hacia mediados del siglo XVI (J. Vargas I68-I69) y su consumo se mantuvo constante durante toda la época colonial.

Si bien en el Nuevo Mundo existía una amplia gama de productos que podían reemplazar el pan de trigo por otros alimentos, como el maíz (tostado o no) (Fernández de Oviedo 596, 598; Saldarriaga, Alimentación 79), había una predilección por este alimento de parte de los españoles, que se encontraba asociada con la costumbre y las ideas que tenían sobre las propiedades nutritivas del trigo (Alzate 24). Se consideraba que el trigo era el "más conveniente al mantenimiento de las gentes que otro pan alguno” y, además, se le atribuían propiedades medicinales en otras preparaciones: podía ayudar a los pulmones, a los problemas de senos endurecidos en madres lactantes, a luchar contra la culebrilla, el catarro y en algunos enfermos tísicos (Herrera I3-I4). La resistencia

II Una de las constataciones interesantes desde la cultura visual, que vale la pena mencionar, es la relevancia otorgada al pan, identificado dentro de la serie de bodegones pintados por Luis Egidio Meléndez para la Corona española entre la décadas de los cincuenta y los setenta del siglo XviII, como uno de los alimentos básicos dentro de la cultura gastronómica española. Basta con observar la centralidad de dos panes redondos en el Bodegón de cocina, que se encuentra en el Museo de Bellas Artes de Asturias, o el rol iconográfico que ocupa en los cuadros Bodegón con ciruelas, brevas, pan, barrilete, jarra y otros recipientes, o Bodegón con peros, queso, pan y recipientes, que se encuentran en el Museo del Prado de Madrid. 
a las variaciones en su dieta se debía a que los nuevos productos americanos no se encontraban inscritos en los códigos culturales alimenticios de los europeos (Saldarriaga, Alimentación 54). La necesidad específica de un producto que culturalmente era considerado insustituible llevó a la disposición de amplias áreas de cultivo en diferentes poblaciones y a la creación de una infraestructura para el procesamiento del trigo en el Nuevo Reino (Satizábal).

Alonso de Zamora, uno de los estudiantes graduados del cust (Plata y Reyes 284), en su Historia de la Provincia de San Antonino del Nuevo Reino de Granada, muestra que el trigo se cultivaba en los valles de tierra fría de las jurisdicciones de Santafé y Tunja; además, presenta una imagen interesante sobre la importancia de este producto en la sociedad neogranadina:

Es tanto el trigo que se coge todos los ańos, que sustentando de pan su numeroso gentio (porque comen pan hasta los Indios) salen cargazones de harina para las Governaciones de Cartagena, Santa Martha, Antiochia, Neyba, y Musso, y otras tierras, en que por ser calidissimas, no se da trigo. También provee de biscocho a los Galeones [...], que todo el ańo entran, y salen de los Puertos del Mar del Norte. Es tierra tan fertil, y propia para el trigo, que muchas vezes se hallan en una caña dos espigas, muy bien granadas. (Zamora 42)

Los datos proporcionados por Zamora coinciden con otra descripción del siglo Xvin que permite hacerse una idea sobre las principales zonas de producción de trigo. Se trata del texto de Basilio Vicente de Oviedo, en el que se encuentran los nombres de los principales lugares de suministro de trigo y harinas: Villa de Leiva, Sáchica, Suta, Ráquira, Tinjacá, Moniquirá, Santo Eccehomo (50), Ubaté (96), Guatavita (97), Sesquilé (IOI), Usaquén (I07), Usme (I08), Paipa (I25), Sátiva (I27) y Soatá (I28). Los niveles de especialización en la producción de trigo en estas zonas eran tan buenos que el religioso consideraba que la calidad era "tan excelente como el de la Andalucía en España" (50).

El segundo alimento en orden de importancia en la alimentación de la comunidad educativa eran las papas o "turmas" ${ }^{12}$. En el periodo analizado, entre I733 y 1737 el consumo de este tubérculo fue de I2 \% del total del gasto, mientras que en $175 \mathrm{I}-\mathrm{I} 755$ se redujo a un $8 \%$. Las turmas eran reconocidas por

I2 Turmas fue una de las palabras usadas por parte de los españoles para nombrar este tubérculo. La explicación sobre las distintas denominaciones que se le ha otorgado a la papa se puede encontrar en el estudio de Diana Bonnet sobre este alimento. 
los españoles americanos como un alimento de "mucho sustento, y de muy buen gusto" (Zamora 42-43); y como lo pone en evidencia Basilio Vicente de Oviedo (96-IO5), se disponía de una gran oferta de este producto en diferentes lugares del altiplano, tales como Ubaté, Guatavita, Chocontá, Cajicá, Sesquilé, Sutatausa y Lenguazaque, entre otros. Un aspecto interesante fue la facilidad de articulación de este alimento en la dieta de los españoles, ya que generó menos resistencia que otros productos originarios de América, como, por ejemplo, el maíz. Se utilizaba de diferentes formas, bien fuera como guarniciones, o bien como ingrediente de algunas preparaciones específicas como el puchero o el ajiaco de papa (J. Vargas I35-I36) 13. En el CusT, para el quinquenio de I733-I737, se compraron en promedio 37,4 cargas de papas al año y en el segundo periodo de análisis, entre I75I y 1755, se adquirieron 22,9 cargas de papa en promedio por año (tabla 3).

$\rightarrow$ TABLA 3 .

Compras de cargas de papas para la alimentación de los colegiales y directivas de la institución entre I733-I737 y I75I-I755

\begin{tabular}{|c|c|}
\hline Año & Cargas \\
\hline I733 & 36 \\
\hline 1734 & 38,5 \\
\hline 1735 & 34 \\
\hline 1736 & 46 \\
\hline I737 & 32,5 \\
\hline Total & $\mathbf{1 8 7}$ \\
\hline
\end{tabular}

\begin{tabular}{|c|c|}
\hline Año & Cargas \\
\hline 1751 & 16 \\
\hline 1752 & 19,5 \\
\hline 1753 & 31 \\
\hline 1754 & 23 \\
\hline 1755 & 25 \\
\hline Total & 114,5 \\
\hline
\end{tabular}

Fuente: elaboración propia con base en AGN, SAA-III, RH-C,I254C

13 En Nueva Granada el puchero contenía los siguientes ingredientes: "agua, pollo, carne de cerdo en trozos, costilla de cerdo, carne de res, costillas de res, carne cecina, longanizas picadas, tocino, plátanos verdes, pelados y partidos en trozos con las manos, plátanos maduros, yuca, papa sabanera, repollo, mazorcas, laurel, ajo, cebolla cabezona, tomillo, sal y pimienta y bastante hogo. Preparación: las carnes se ponen a cocinar con los condimentos hasta que estén tiernas, se sacan y se añaden al caldo, el plátano y las mazorcas, luego las Papas, la yuca y el repollo y se dejan hervir. El plátano maduro se hace aparte con panela. Se escurre todo y se sirve en cada plato una porción con cada ingrediente y se baña con el hogo caliente" (Flórez y Ortiz, cit. en Restrepo 87). 
Otro de los alimentos que consumían los colegiales y las directivas de la institución era el arroz. Este cereal, que tiene una densidad energética igual a la del maíz y mayor que la del trigo, solo es superado por otros alimentos ancestrales americanos como el amaranto o la chía ${ }^{\mathrm{I4}}$. De hecho, su consumo en la Colonia también se encontraba asociado con la idea de ser un alimento con propiedades medicinales (Alzate 32). A juzgar por lo que sostiene Pedro Fermín de Vargas (I33-I36), este alimento podía consumirse en el siglo XVIII de diferentes formas, como acompañamiento, colada, en un puchero o guisado, y su porción por persona podría variar entre una y tres onzas. Los principales lugares de producción de este cereal se encontraban en Simacota, Neiva e Ibagué (Oviedo 49, 240 y 249).

En cuanto a la proteína, el consumo de aquella de origen animal fue más elevado que el de los alimentos del mundo vegetal como frijoles o garbanzos. En la lista de compra del cust se encontró que el consumo cotidiano de cecina y jamones de cerdo se realizaba semanalmente. De la primera se compraba entre media y una arroba, en tanto que de la segunda se adquiría una unidad de jamón (AGN, SAA-III, $R H-C$, I254C). En la lista no se especifica qué tipo de carnes y de qué manera se consumían, aunque es claro que se trata de carne de res y de carnero. En Santafé había mucho ganado vacuno y la ciudad era abastecida desde distintos puntos, ya fuera desde los Llanos Orientales o desde Neiva (Saldarriaga, "Consumo"; Castaño).

El suministro de sal para la ciudad de Santafé fue mucho más estable que para otras regiones, lo que se explica por la cercanía de Zipaquirá y la abundancia de este producto puesto en los mercados adyacentes, tanto en la región del altiplano como en el nororiente del virreinato (Ocaña, Socorro, Pamplona, entre otros). En nuestro análisis, el suministro de sal fue constante, a un precio aproximado de 3,5 reales la arroba, lo cual representó ventajas para su consumo en el cust. Otros productos incluidos en la canasta básica de la institución fueron conservas de dulce, arvejas y ajos. Sin embargo, las cantidades compradas de estos productos se desconocen en su totalidad por el tipo de registro elaborado por los escribanos de la institución.

I4 Información proporcionada por el cuadro de "Densidades energéticas de algunos granos (k/cal/roo): maíz 365, arroz 365, trigo 327, amaranto 37 y yía 490” (Pureco 28). 


\section{Las compras de alimentos en el Colegio Santo Tomás de Aquino. Entre la cultura y el mercado}

El transcurso de la conquista y el establecimiento de los españoles en Santafé se circunscribe al proceso denominado "primera globalización", que conectaría las regiones "densamente pobladas directa o indirectamente, de forma tal que quedarían vinculadas profunda y permanentemente” (Flynn y Giraldez 3I). Este proceso se caracterizó por la expansión económica y cultural de las potencias europeas, a la que se integraron Asia, América, Europa y África mediante distintos procesos socioeconómicos y culturales. Como bien lo señalan Romero y Pérez:

Los elementos culturales de un continente se implantaron en otro; las enfermedades y las plantas medicinales, así como los cultígenos (trigo, maíz, papa, frutales) y animales domésticos, se empezaron a introducir y a adaptar a los nuevos ambientes. (II)

En este contexto, varias de las razones de consumo de la institución se encuentran vinculadas a las posibilidades del territorio por medio de los productos ancestrales originarios americanos (papas, sal, pescados locales, arracacha, frijoles, ají, tomate, cacao, entre otros), las transformaciones ecológicas de escala mundial que introdujeron los españoles (cultivo del trigo, domesticación de ganado bovino y porcino, garbanzos, arroz, azúcar, habas, arroz, entre otros), las restricciones o regulaciones establecidas por las creencias religiosas de la orden y, por supuesto, las posibilidades del mercado. Esta composición sintetizaría las posibilidades de un sincretismo gastronómico a partir de un mestizaje alimenticio en el CUST desde su fundación, permitiendo la preparación de diferentes platos en el contexto regido por la orden, como gigote de gallina, guisados, asados y pucheros (Restrepo 70).

Según los registros del libro de gasto diario, uno de los consumos con mayor regulación, debido a la doctrina religiosa, fue el de carne. Cecilia Restrepo ha mostrado, por medio del estudio de las Constituciones utilizadas por los dominicos, cómo había prohibiciones al consumo de carne en los días de cuaresma y, citando a Toussaint-Samat, sugiere que inclusive se podría hablar de unos I8o días al año sin consumo de carne por parte de los colegiales (7I). En el caso del Cust, aunque no se pudo constatar la afirmación de ToussaintSamat, se encontró que la comunidad se abstuvo del consumo de carne durante 
setenta días, aproximadamente, fechas vinculadas con la cuaresma, la Semana Santa, témporas, más de una decena de vigilias y la celebración de la Natividad. Durante estas celebraciones se incrementaba el consumo de pescado, mientras que en las vigilias y las témporas se reemplazaban las proteínas bovinas y porcinas por mayores compras de leche y huevos o la preparación de sopas. Algunos de los registros ponen en evidencia la sustitución de la carne por otros alimentos, por ejemplo, en la tercera semana de junio de 1735 se anotó: "Dimos quatro r[eale]s p[ar]a guebos, y leche p[ar]a la Vigilia de S[a]n Juan", mientras que en septiembre de ese mismo año se destinaban los mismos rubros pero para dos celebraciones religiosas adicionales: "Dimos ocho r[eale]s p[ar]a la Vigilia de S[a]n Matheo, y otro día de Temporas, guebos, leche" (AGN, SAA-III, $R H-C$, I254C, ff. I2 r.-v.). Las razones por las cuales no se consumían carnes rojas se relacionan con ideas sobre la purificación del espíritu (tabla 4).

$\rightarrow$ TABLA 4

Celebraciones religiosas del Colegio y Universidad de Santo Tomás entre I733-I737 y I75I-I755

\begin{tabular}{|c|c|c|}
\hline \multicolumn{2}{|c|}{ Fecha/duración } & \multirow{2}{*}{ Festividades religiosas } \\
\hline Día & Mes & \\
\hline \multicolumn{2}{|c|}{40 días } & Cuaresma \\
\hline \multicolumn{2}{|c|}{7 días } & Semana Santa \\
\hline \multicolumn{2}{|c|}{ I2 días } & Témporas de la Santa Cruz \\
\hline 23 & \multirow{2}{*}{ Junio } & Vigilia de la Natividad de San Juan Bautista \\
\hline 28 & & Vigilia de San Pedro y San Pablo \\
\hline 24 & Julio & Vigilia de Santiago \\
\hline 9 & \multirow{3}{*}{ Agosto } & Vigilia de San Lorenzo \\
\hline I 4 & & Vigilia de la Asunción de la Virgen \\
\hline 23 & & Vigilia de San Bartolomé \\
\hline 20 & Septiembre & Vigilia de San Mateo \\
\hline
\end{tabular}




\begin{tabular}{|c|l|l|}
\multicolumn{2}{|c|}{ Fecha/duración } & \multicolumn{2}{|}{ Festividades religiosas } \\
\hline Día & \multicolumn{1}{|c|}{ Mes } & \\
\hline 27 & \multirow{2}{*}{ Octubre } & Vigilia de San Simón \\
& & Vigilia de todos los Santos \\
\hline 31 & Noviembre & Vigilia de San Andrés \\
\hline 29 & \multirow{2}{*}{20} & Vigilia de Santo Tomás \\
\hline 24 & & Vigilia de la Navidad \\
\hline
\end{tabular}

Fuente: elaboración propia con base en AGN, SAA-III, RH-C, I254C

Por otra parte, también es importante indagar sobre otro tipo de decisiones alimenticias que se tomaron en la comunidad y que escapan al mundo doctrinal relacionado con el consumo. Nos referimos a la racionalidad económica de los dominicos al adquirir productos que se encontraban con costos bajos o elevados en el mercado. Como se puso en evidencia mediante gráficas de composición del gasto (véase figuras 2 y 3), la dieta del cust era alta en carbohidratos y, después del pan, el segundo alimento de mayor importancia fue la papa. El artículo de Bonnett muestra cómo desde muy temprano en el siglo xvi este alimento ancestral originario fue incorporado por sus altos niveles nutricionales a la dieta de los españoles peninsulares y americanos, de diferentes maneras (39-42), y las cifras de cargas consumidas en el CUST confirman su importancia.

Para responder a esta inquietud hemos analizado la evolución del consumo de papa en función de los precios y las cantidades del producto en los dos quinquenios. Por tratarse de uno de los alimentos de la canasta básica del consumo santafereño en el siglo XVIII, consideramos que constituye una buena muestra del comportamiento económico de los dominicos frente al mercado (figura 4).

Al observar la representación gráfica (figura 4), donde el eje horizontal lo constituyen los cuarenta trimestres de los dos quinquenios analizados, el primer eje vertical las cantidades de cargas consumidas y el segundo eje vertical los precios, se puede encontrar una correlación negativa entre precios y cantidades (Pearson $=0,42$, lo cual indica que existe una relación inversa en la cual el consumo de papa es elástico al precio; es decir, cuando había un aumento de precio se reducía el consumo de este bien. En la figura 4 se pueden identificar dos coyunturas con cinco periodos muy claros en esta relación inversa. La 
primera, cuando los precios de las cargas de papa eran bajos, lo cual aumentó su consumo en 1736 (trimestre 9) y I753 (trimestre 3I), y la segunda, cuando el precio de la papa subía y por ende el consumo del bien bajaba, lo que se puede ver para los años de 1735 (trimestre 9), I75I (trimestre 2I) y I752 (trimestre 25). Este comportamiento observado en los datos del libro de gasto diario del cust pone en evidencia una racionalidad económica de los agentes muy pendiente de los movimientos del mercado, lo cual resulta interesante pues los dominicos utilizaron como sustituto incrementar las cantidades de compra de productos como el garbanzo o el arroz cuando se presentaba el encarecimiento del tubérculo. Lo que se puede deducir de las decisiones tomadas con relación a estas dos muestras de alimentos como la carne y la papa es la coexistencia de los motivos económicos y culturales que impulsaron la manutención de la institución en esos dos quinquenios.

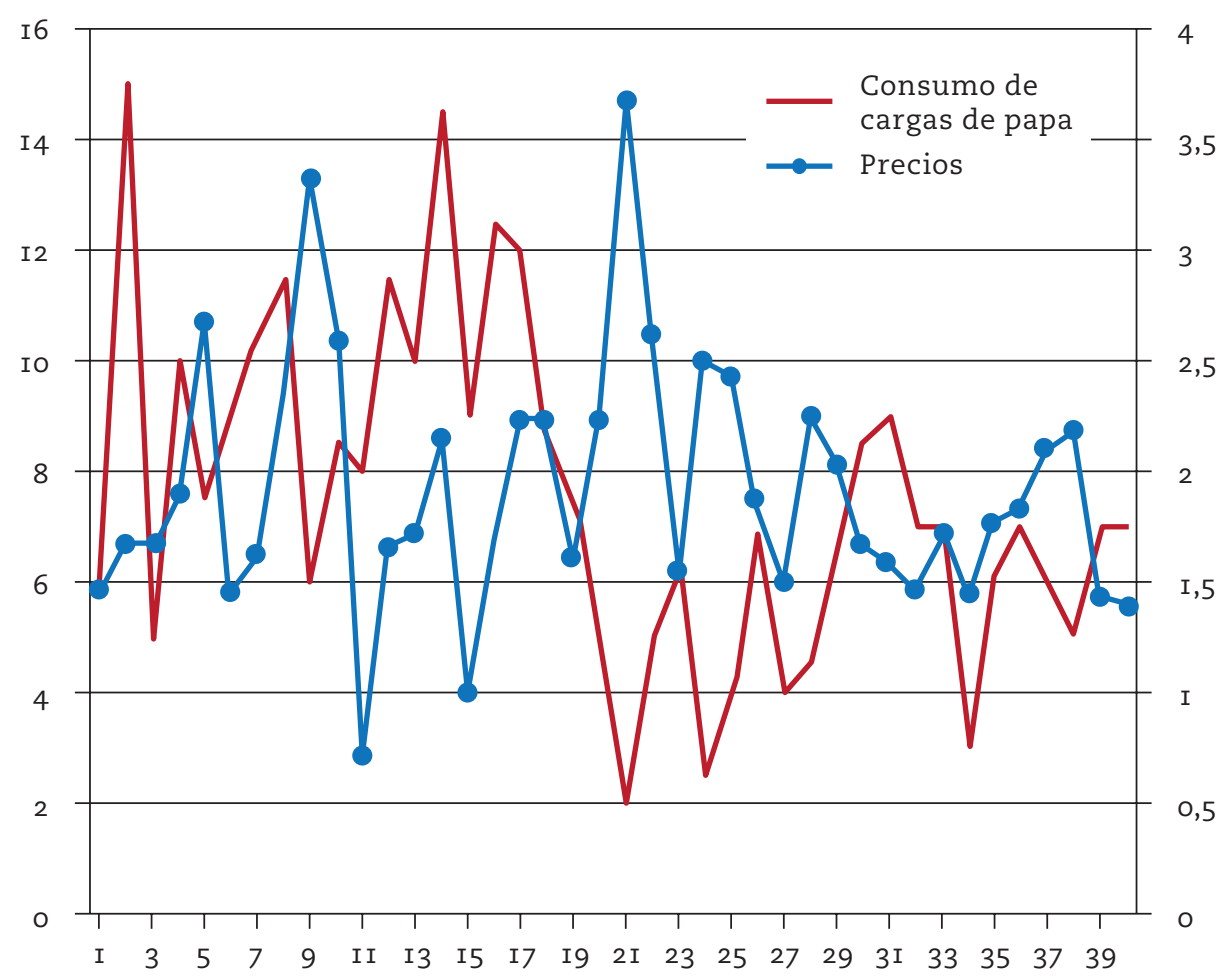

or FIGURA 4 .

Relación entre los precios y las cantidades de papa consumidas por el Colegio y Universidad Santo Tomás de Aquino, I733-1737 y I75I-I755

Fuente: elaboración propia con base en AGN, SAA-III, RH-C, I254C 


\section{Vino y aceite. Los productos garantizados por el rey}

Uno de los productos fundamentales en la dieta de los españoles fue el vino, cuyo consumo era característico de la cultura gastronómica y medicinal europea. Fuera del ámbito religioso, se lo consideraba una bebida agradable, estimulante y saludable si se consumía con moderación (Herrera 45). El vino fue considerado por los españoles un bien fundamental, no solo para el consumo recreacional o alimenticio, sino por el marco de su universo de creencias médicas (J. Vargas I89; Alzate 34). Tanto el trigo como el vino eran los alimentos centrales de la celebración católica más importante, el sacramento de la eucaristía. En esta el ritual simbólico representaba la recepción del cuerpo y la sangre de Cristo, encarnados en el pan y el vino que consagraban el símbolo de la fe de la sociedad cristiana, donde de forma ritual cada vez que se realizaba una misa se anunciaba la muerte de Jesucristo y se proclamaba su resurrección hasta su regreso (Earle 699).

La figura administrativa que se creó para el suministro de los productos más costosos del sacramento fue la donación, conocida entonces como las mercedes de limosnas de vino, cera y aceite ${ }^{\text {I5 }}$. Estas limosnas fueron otorgadas entre el siglo XVI y el XVIII a las distintas órdenes religiosas, conventos y sacerdotes en todos los reinos de ultramar de la Corona española ${ }^{16}$. Por lo general, los documentos eran reales cédulas que ordenaban a los oficiales de la Real Hacienda proveer por un tiempo determinado — que convencionalmente siempre era prorrogado a solicitud de las órdenes religiosas - de "vino y azeyte para con que pudiesen celebrar y alumbrar el Sanctisimo Sacramento [...] que fuese necesario para una lámpara y a razón de arroba y media de vino en cada un año" (AGN, C, $M$, t. 67, f. 357 r.).

La historiografía ha mostrado que el consumo del vino era costoso, excepto para algunos individuos que se encontraban en el marco de las tempranas economías extractivas del siglo XVI y XVII, como la pesquería de perlas en Santa

I5 Las regulaciones principales se establecieron desde muy temprano en la Recopilación de leyes de los Reinos de las Indias. Mandadas imprimir y publicar por S.M.C. del Rey Don Carlos II, cuyas leyes VII a IX explicaban las regulaciones de la limosna de vino y aceite, que se debía dar en dinero o especie de vino y aceite, solo a religiosos conventuales y de forma regulada con moderación (I3).

I6 En el Archivo General de Indias se encuentra una gran cantidad de expedientes sobre las limosnas de vino y aceite que fueron otorgadas a distintas comunidades religiosas en todas las posesiones de ultramar entre los siglos XVI y XVIII (AGI, $S G, A S$, t. 963; AGI, $S G, A F$, t. 318; AGI, $S G, A S D$, t. 267I; AGI, $S G, A G$, t. 225; AGI, $S G, A B A$, t. 594). 
Marta, la minería aurífera en "Zaragoza, Cáceres y Guamocó en Antioquia”, o de los circuitos de contrabando extranjero en Río de la Hacha (Saldarriaga, Alimentación 63-65, 104). De manera paralela, se ha puesto en evidencia que en los primeros siglos, debido a sus bajos impuestos y al mismo tipo de economía extractiva, el consumo de vino se había convertido en parte central de la mesa de algunos santafereños, siendo inclusive uno de los principales productos consumidos por algunos habitantes de la ciudad (J. Vargas I53, I59 y I67). Sin embargo, desde las primeras décadas del siglo Xviı y a partir de los aranceles que se impusieron a los vinos y los aceites provenientes de Sevilla y Cádiz, su consumo presentó una disminución importante y se hizo una sustitución de productos en parte de la sociedad santafereña, sin abandonárseles completamente (Cruz 28 y 48). En el marco de las órdenes religiosas, el consumo era principalmente determinado por las jerarquías eclesiásticas, aunque algunos padres "achacosos y ancianos' acompañaban sus comidas con algo de vino", lo cierto es que "el vino era una exclusividad del ritual de la misa y de los clérigos [...]” (Pérez 83).

Se puede ver que en los colegios administrados por la Orden de Predicadores había un abastecimiento de vino y aceite por parte de las mercedes de limosnas de estos dos productos. Sin embargo, en las constituciones no se pudo constatar que a los colegiales se les incluyera dentro de su dieta de manera regular (AHUR, $C, 4$, f. 3 v.), excepto obviamente mediante la regularidad del sacramento o algún uso medicinal.

Igualmente, se puede establecer que en la alimentación del cust el uso del aceite era restringido. Este era un producto que se encontraba en la institución gracias a las mercedes de limosnas (Zamora 529) y a las compras realizadas por los padres dominicos, que generalmente ingresaba como un gasto extraordinario (AGN, SAA-III, $R H-C$, I254C, ff. I7I v. y I79 r.). En el análisis sobre el bajo nivel de consumo de aceite de oliva dentro de la institución, se encuentra que el problema era su alto costo por ser un bien importado. Aunque en Villa de Leiva había olivares que daban excelentes aceitunas para la fabricación de este producto, la elaboración local estaba prohibida por la Corona (Zamora 42). Podemos ver entonces cómo con el aceite sucedía lo mismo que con el vino: un marco de protección de la industria de la metrópoli que restringió la posibilidad de generar una producción local. Por esta razón, en el Nuevo Reino de Granada la manteca de cerdo pasó a ser el medio de fritura e ingrediente esencial desde el siglo XVI (J. Vargas 195) hasta el XVIII. En el cust, por ejemplo, en el periodo I733-I737 hubo un promedio de consumo anual de diez arrobas anuales, es decir, aproximadamente $9,5 \mathrm{~kg}$ mensuales, siendo el quinto gasto más significativo. 
Durante el siguiente quinquenio, I75I-I755, el consumo promedio anual fue de ocho arrobas, es decir 7,5 kg mensuales, siendo la cuarta inversión de mayor importancia para el periodo (AGN, SAA-III, $R H-C$, I254C).

Finalmente, es importante señalar que hubo otros productos fundamentales dentro de la dieta de las comunidades religiosas de Santafé, como gallina, quesos, miel, algunas hortalizas y frutas que no necesariamente pasaban por los registros del libro de gastos de la institución. Estos productos probablemente eran suministrados al cust por vía de las haciendas de la Orden de Predicadores $^{17}$, tal y como se realizaba en su homólogo de Nuestra Señora del Rosario, donde se suministraban varios de los productos provenientes de las haciendas: San Vicente, El Tejar de las Nieves y Calandayma (Restrepo 65-67). Sin embargo, no se han podido encontrar los acervos documentales que permitan caracterizar los productos y las respectivas cantidades que eran enviadas desde las haciendas a la institución.

\section{Notas finales}

El análisis del libro de gasto ordinario del cust ha permitido conocer parte de la dieta de una de las instituciones educativas religiosas más importantes del Nuevo Reino de Granada, así como también la periodicidad y la programación de la compra de alimentos. La mayoría de los productos son característicos del altiplano o sus inmediaciones, tales como la leña, las habas, las turmas y las arracachas. La comunidad educativa estaba acostumbrada a consumir productos básicamente regionales, por lo menos en un $65 \%$ del total.

Una de las características interesantes de la contabilidad de los dominicos es la marcada relación existente entre las festividades religiosas y el tipo de alimentos comprados para la comunidad educativa. Por ejemplo, cada ańo, en la época de Semana Santa incrementaba el gasto en pescado (AGN, SAA-III, $R H-C$, I254C, ff. 98 r.- I75 r.). Al mismo tiempo, se puede observar en los libros de contabilidad un descenso en el consumo de carnes rojas (jamón, lomo, costillas de cerdo, cecina).

I7 Una de las haciendas que se referencian con frecuencia es La Huerta, que se encontraba en Tocaima. Sin embargo, no se ha encontrado información económica de ella, más allá de su valor y forma de adquisición (Barrado 228). 
Sin duda, puede parecer un hecho de menor importancia si se piensa que, en general, la sociedad colonial era de tipo confesional. Sin embargo, si se analiza desde el punto de vista de las relaciones sociales de la construcción de una comunidad "académico-religiosa", servía como un vehículo efectivo de la reproducción de la sociedad, más aún si se piensa, como lo plantearían Mary Douglas y Baron C. Isherwood con relación a los significados públicos del consumo (8o). Finalmente, la alimentación de la comunidad, que se hacía en los refectorios de la institución, reafirmaba procesos de identidad, donde sin estar necesariamente en presencia de un sermón o liturgia específica, el simple acto de consumir determinados alimentos o estar restringido de acceder a otros - directrices supervisadas por el reverendo padre rector - tenía implícito un contenido simbólico ritual más allá de los estudios colegiales-universitarios. En última instancia, el acto de comer, más allá de la necesidad biológica en sí misma, también representaba una síntesis que pone de manifiesto el entramado cultural de la sociedad eclesiástica, sus posibilidades ecológicas y sus determinantes económicas. La inversión en alimentos para el consumo institucional generó relaciones sociales "rituales" en torno al consumo. En tal sentido, este tipo de consumo "básico", más allá de relaciones como la emulación (Veblen), dio lugar a un afianzamiento y una reproducción del orden social, que fue indirecto mediante la compra y el suministro de alimentos.

Para finalizar, un elemento de interés particular con respecto al consumo del cust es el bajo consumo de artículos de lujo (de los llamados efectos de Castilla), pues para la época se conoce la predilección por el vino y otros bienes importados. Si bien se ha constatado que había un suministro constante de vino mediante las limosnas de vino y aceite otorgadas por la Corona para el servicio religioso de la orden, no se registran compras adicionales para el consumo frecuente en la institución. De acuerdo con la historiografía (Pérez 83) y con fuentes analizadas (AHUR, $C, 4$, f. 3 v.), este hecho lleva a considerar que su consumo estaba limitado a las jerarquías más altas y no al colegiado en general. Esto es importante tenerlo en cuenta, ya que ocurre algo completamente diferente en una región como Popayán, donde debido al dinámico comercio que mantenía con Quito y la región aurífera, y la diferencia del tipo de población española asentada en el sur del virreinato y su capacidad adquisitiva, fue posible la adquisición de más botijas de vino, además de las que eran otorgadas por la Corona (ACC, OS, 9609). Una hipótesis adicional podría poner en evidencia que fuera de los factores de composición religiosa y de los factores geográfico-espaciales de los recursos, también hay una variable demográfica fuerte que diferencia los 
gustos y las orientaciones del consumo, tal como lo plantearía Pierre Bourdieu en La distinción (1979).

\section{6 \\ B I B L I O G R A F í A}

\section{FUENTES PRIMARIAS}

\section{A. Archivos}

Archivo General de Indias, Sevilla (AGI)

Sección Gobierno (sG)

Audiencia de Buenos Aires (ABA), tomo 963.

Audiencia de Filipinas $(A F)$, tomo 318.

Audiencia de Guadalajara $(A G)$, tomo 225.

Audiencia de Santafé (AS), tomo 963.

Audiencia de Santo Domingo (ASD), tomo 2671.

Archivo Histórico de la Universidad del Rosario (AHUR)

Constituciones $(C) 4$

"Expediente presentado por Juan Peláez con la cuenta de gastos e ingresos durante su Rectorado". Caja 2.

Archivo General de la Nación, Bogotá, Colombia (AGN)

Sección Colonia (c)

Colegios $(C), \mathrm{I}$.

Miscelánea $(M), 67,7 \mathrm{I}$.

Sección Archivo Anexo III (SAA-III)

Real Hacienda - Cuentas $(R H-C)$ I254c.

Archivo Central del Cauca, Popayán (ACC)

Ordenes Sagradas (OS) 9503, 9504, 9453, 9454, 9609, 9610, 9602, 9600, 9599, 9598, 9590, 9591, 9592, 9594.

Archivo Histórico de Antioquia, Medellín (AнA)

Colonia (C) 457-458. 


\section{B. Impresos}

Diccionario de la Real Academia Española de la Lengua (DRAE). Madrid: Real Academia Española de la Lengua, 1734 .

Fernández de Oviedo, Gonzalo. Historia general y natural de las Indias. Islas y Tierra-Firme del mar Océano. T. Iv. Madrid: Imprenta de la Real Academia de la Historia, I855.

Herrera, Gabriel Alonso de, ed. Agricultura general del campo. Madrid: Domingo Gonçalez, i6ig.

Oviedo, Basilio Vicente de. Riquezas y cualidades del Nuevo Reino de Granada. Bogotá: Imprenta Nacional, I930.

Recopilación de leyes de los Reinos de las Indias. Mandadas imprimir y publicar por la Magestad Católica del Rey Don Carlos II. Tomo I. Madrid: Boix Editor, I84I.

Vargas, Pedro Fermín de. Pensamientos politicos y memoria sobre la población del Nuevo Reino de Granada. Bogotá: Biblioteca Popular de Cultura Colombiana, 1944.

Zamora, fray Alonso de. Historia de la provincia de San Antonino del Nuevo Reino de Granada. Bogotá: Instituto Colombiano de Cultura Hispánica, 1980.

\section{Cuadros referenciados}

\section{Museo de Bellas Artes de Asturias, Oviedo (España)}

Colección Pedro Masaveu

Meléndez, Luis Egidio. Bodegón de cocina. 1772. http://www.museobbaa.com/ obra/bodegon-de-cocina/

\section{Museo del Prado, Madrid (España)}

Serie Bodegones para el Gabinete de Historia Natural del Príncipe de Asturias (futuro Carlos IV)

Meléndez, Luis Egidio. Bodegón con ciruelas, brevas, pan, barrilete, jarra y otros recipientes. $1760-1770$. https://www.museodelprado.es/coleccion/obra-de-arte/ bodegon-con-ciruelas-brevas-pan-barrilete-jarra-y/ee74dc9d-7c8e-45e6-8ab88 cae8do835ai -.-. Bodegón con peros, queso, pan y recipientes. Tercer cuarto siglo XviII. https:// www.museodelprado.es/coleccion/obra-de-arte/bodegon-con-peros-queso-pan-yrecipientes/4a039125-069f-4990-94c7-eribasf6d6c2 


\section{I. FU E N T ESSECUNDAR I A S}

Andrés Ucendo, José Ignacio y Ramón Lanza García. “El abasto de pan en el Madrid del siglo XVII". Studia Historica: Historia Moderna, vol. 34, 2012, pp. 6I-97.

Alzate Echeverri, Adriana María. “Comer en el hospital colonial: apuntes sobre la alimentación en tres hospitales neogranadinos a finales del siglo XviII". Historia Crítica, n. 46, 2012, pp. 18-42. DOI: https://doi.org/10.7440/histcrit46.2012.03.

Amaya, José Antonio y James Vladimir Torres Moreno. “La Expedición Botánica a la luz de las cifras, 1783-1816”. Ensamblando Estados, editado por Olga Restrepo Forero. Bogotá: Universidad Nacional de Colombia, Facultad de Ciencias Humanas, Centro de Estudios Sociales (CES), 20I3, pp. 4I-75.

Barrado Barquilla, José, ed. Los dominicos y el Nuevo Mundo, siglos XVIII-XIX. Salamanca: Editorial San Esteban, 1995.

Bernadot, Vicente María. La orden de predicadores. Bogotá: Editorial A. B. C., 1948.

Bonnett, Diana. “'Resurgencia’ y recolonización de la papa. Del mundo andino al escenario alimentario mundial, siglos XVI-XX”. Anuario Colombiano de Historia Social y de la Cultura, vol. 46, n. ${ }^{\circ}$ I, 2019 , pp. 27-57. DoI: https://doi.org/10.15446/achsc.v46ni.75552.

Bourdieu, Pierre. La distinción: criterios y bases sociales del gusto. Madrid: Taurus, 2006.

Castaño Pareja, Yoer Javier. "Consumo y abasto de la carne en la ciudad de Santafé del Nuevo Reino de Granada, 1572-1716”. Fronteras de la Historia, vol. 22, n. ${ }^{\circ}$ 2, 2017, pp. 76 -113. DOI: https://doi.org/10.22380/20274688.105.

Cruz Medina, Juan Pablo. “Alimento, mestizaje y cultura. Una aproximación a la historia de la alimentación en la Santafé del siglo XviI”. Boletín Museo del Oro, n’. 57, 2017, pp. 4-57.

Douglas, Mary y Baron C. Isherwood. El mundo de los bienes. Hacia una antropología del consumo. México D. F.: Editorial Grijalbo, 1990.

Earle, Rebecca. “'If You Eat Their Food...': Diets and Bodies in Early Colonial Spanish America”. The American Historical Review, vol. II5, n. ${ }^{\circ}$ 3, 2010, pp. 688-713. DoI: https:// doi.org/ıo.1086/ahr.II5.3.688.

Flynn, Dennis y Arturo Giraldez. "Los orígenes de la globalización en el siglo Xvi”. Oro y plata en los inicios de la economía global, coordinado por Bernd Hausberger y Antonio Ibarra. México: Colegio de México, 2014, pp. 29-76.

Frid, Carina. "Precios y crisis en una economía rioplatense. Santa Fe (1790-1850)". América Latina en la Historia Económica, vol. 24, n. ${ }^{\circ}$ 2, 2017, pp. 59-92, DOI: https://doi. org/10.28232/alhe.v24i2.746. 
Llopis Agelán, Enrique, Alfredo García-Hiernaux, Héctor García Montero, Manuel González Mariscal y Ricardo Hernández García. "Índices de precios de tres ciudades españolas, 1680-1800: Palencia, Madrid y Sevilla”. América Latina en la Historia Económica, n. ${ }^{\circ}$ 32, 2009, pp. 31-80. DOI: https://doi.org/10.18232/alhe.vi6i2.416.

Pacheco, Juan Manuel. Historia eclesiástica. T. 2. La consolidación de la Iglesia, siglo XVIII. Bogotá: Academia Colombiana de Historia, Lerner, Historia Extensa de Colombia, vol. XIII, I975.

Pérez Morales, Edgardo. "Vida material, religiosidad y sociedad colonial. Espacios, objetos y prácticas de consumo en el Colegio jesuita de la ciudad de Antioquia, 1726-1767". Historia Critica, n. ${ }^{3}$ 8, 2009, pp. 70-95. DOI: https://doi.org/I0.7440/histcrit38.2009.05.

Plata Quezada, William Elvis y Norma Constanza Reyes Escobar. La Universidad Santo Tomás de Colombia ante su historia, siglos XVI-XIX. Bogotá: Universidad Santo Tomás, 2005 .

Pureco Ornelas, José Alfredo. "El maíz de México para el mundo. Alimento, patrimonio y ese 'oscuro objeto del deseo'”. El pasado del futuro alimentario: los alimentos ancestrales americanos, editado por Enriqueta Quiroz y Helena Pradilla Rueda. México: Instituto de Investigaciones Dr. José María Luis Mora, 20I8, pp. 2I-4I.

Real Academia Española. Diccionario de la lengua española, 23. ${ }^{\mathrm{a}}$ ed. Actualización 2020. http://dle.rae.es

Restrepo Manrique, Cecilia. La alimentación en la vida cotidiana del Colegio Mayor de Nuestra Señora del Rosario, I653-1773. Bogotá: Editorial Universidad del Rosario, 2005.

Romero, Luis René y Juan Felipe Pérez. Naufragios y puertos maritimos en el Caribe colombiano (siglos XVI-XVIII). Bogotá: Siglo XXI Editores, 2005.

Saldarriaga, Gregorio. Alimentación e identidades en el Nuevo Reino de Granada, siglos XVI y XVII. Bogotá: Ministerio de Cultura, 2012. DOI: https://doi.org/I0.I215/001821682802726 .

---. "Consumo de carnes en zonas cálidas del Nuevo Reino de Granada: cualidades cambiantes, siglos XVI y XVII". Fronteras de la Historia, vol. II, 2006, pp. 2I-56. DoI: https:// doi.org/10.22380/20274688.513.

Satizábal Villegas, Andrés Eduardo. Molinos de trigo en la Nueva Granada, siglos XVIIXVIII. Arquitectura industrial. Patrimonio cultural inmueble. Bogotá: Universidad Nacional de Colombia, 2004.

Torres Moreno, James Vladimir. "El comportamiento de los precios en una economía preindustrial: Popayán, virreinato de Nueva Granada, 1706-1819”. Cuadernos de Economía, vol. 34, n. ${ }^{\circ}$ 66, 2015, pp. 629-680. DoI: https://doi.org/10.15446/cuad.econ.v34n66.506II. 
---. Minería y moneda en el Nuevo Reino de Granada. El desempeño económico en la segunda mitad del siglo XVIII. Bogotá: ICANH, 2013.

Vargas Lesmes, Julián. La sociedad de Santafé colonial. Bogotá: Cinep, 1990.

Veblen, Thorstein. La teoría de la clase ociosa. México: Fondo de Cultura Económica, 2004.

Zambrano Pantoja, Fabio. Alimentos para la ciudad. Historia de la agricultura colombiana. Bogotá: Editorial Planeta, 2015. 